\title{
Recovery of the old nova V840 Ophiuchi: A carbon rich system ${ }^{\star}$
}

\author{
L. Schmidtobreick ${ }^{1}$, C. Tappert ${ }^{2}$, A. Bianchini ${ }^{3}$, and R. E. Mennickent ${ }^{2}$ \\ ${ }^{1}$ European Southern Observatory, Casilla 19001, Santiago 19, Chile \\ 2 Grupo de Astronomía, Universidad de Concepción, Casilla 160-C, Concepción, Chile \\ 3 Dipartimento di Astronomia, Università di Padova, Vicolo dell'Osservatorio 2, 35122 Padova, Italy
}

Received 27 May 2003 / Accepted 18 August 2003

\begin{abstract}
We present optical spectroscopy and multi colour photometry of the old nova V840 Oph. We rediscovered the nova based on its position in the colour-colour diagrams. It stands out as a very blue object with an additional red component. We present the first optical spectroscopy of this candidate and confirm its nova character. Furthermore, V840 Oph has been found as one of very few cataclysmic variables showing C IV emission at $\lambda=580 / 1 \mathrm{~nm}$. From the analysis of the carbon lines it seems probable that V840 Oph contains actually a carbon-rich secondary star. So far, only the nova-like QU Car has been known to have such a companion. We furthermore find spectroscopic evidence that V840 Oph has a hot, dense accretion disc or stream and is probably a magnetic system.
\end{abstract}

Key words. stars: novae, cataclysmic variables - stars: individual: V840 Oph

\section{Introduction}

V840 Oph, a classical Galactic nova, has first been visible as a star of 6.5 mag on Harvard photographs taken in May 1917 (Bailey 1920). It has been a fast nova with two very distinct secondary maxima (Shapley 1921), and has been classified by Duerbeck (1981) as Bb (decline with major fluctuations). He also gave the value for $t_{3}=36 \mathrm{~d}$ which places V840 Oph among the fast novae. Three stars are close to the position of the nova and possible candidates for the nova remnant (Duerbeck 1987).

Several surveys have attempted to identify V840 Oph, but without much success. No detection has been accomplished in the 2 mass second incremental data release (Hoard et al. 2002). Munari \& Zwitter (1998) performed spectrophotometric measurements for 20 faint cataclysmic variables but V840 Oph has been too faint to be included. Harrison \& Gehrz (1994) have observed several novae in four IR bands with IRAS and obtained a detection of V840 Oph at $25 \mu \mathrm{m}$. However, due to the large FWHM of the IRAS signal (the uncertainty of the coordinates is 0.5 for $25 \mu \mathrm{m}$ ) source confusion is very likely, and no unambiguous identification can be achieved in this crowded field.

In the course of a long-term project investigating novae with large outburst amplitudes, we have performed multi wavelength photometry of the stars in the field of V840 Oph and in particular of the three candidates for the nova. The aim was to recover the nova via its colour characteristics and to confirm it

Send offprint requests to: L. Schmidtobreick,

e-mail: 1schmidt@eso.org

* Based on observations collected at the European Southern Observatory, La Silla, Chile. spectroscopically. In a further investigation of the system, we also analyse its spectroscopic peculiarities.

\section{Observation and data reduction}

The photometric data were obtained in one night on 2001-07-15 at the $1.54 \mathrm{~m}$ Danish telescope at ESO, La Silla, using DFOSC. Spectroscopic observations were performed on 2002-04-20 and on 2003-02-28 using EFOSC2 at the $3.6 \mathrm{~m}$ telescope at La Silla. In Table 1 the observational parameters are summarised.

The standard reduction of the photometric data including BIAS subtraction and flatfielding have been performed using IRAF. For the stellar photometry, the standalone packages of DAOPHOT and ALLFRAME have been used to perform aperture and PSF photometry of all stars in the observed field. Only those stars with photometric uncertainties below 0.03 mag have been selected for further analysis. Photometric calibration has been obtained with MIDAS using several standard stars selected from Landolt (1992).

The reduction of the spectroscopic data has been done with IRAF only. The BIAS has been subtracted and the data have been divided by a flat field, which was normalised by fitting Chebyshev functions of high order. The spectra have been optimally extracted (Horne 1986). Wavelength calibration yielded a final resolution of $0.92 \mathrm{~nm} F W H M$ for the 2002 data and $1.63 \mathrm{~nm}$ FWHM for the 2003 data. Flux calibration was performed only for the 2003 spectrum, using the spectrophotometric standard LTT 6248 which has been observed directly after the object with an airmass difference of 0.1 . The photometric error has been derived by comparing the three 
Table 1. All observations obtained for this research are listed with their date, the instrument/telescope combination, the used filter or grism and slit width, and the exposure time.

\begin{tabular}{cccc}
\hline \hline Date & Instrument & Filter or Grism/Slit & Exp-time [s] \\
\hline 2001-07-15 & DFOSC/1.54D & $U$ & 900 \\
$2001-07-15$ & DFOSC/1.54D & $B$ & 900 \\
$2001-07-15$ & DFOSC/1.54D & $V$ & 600 \\
$2001-07-15$ & DFOSC/1.54D & $R$ & 900 \\
$2001-07-15$ & DFOSC/1.54D & $I$ & 900 \\
$2002-04-20$ & EFOSC/3.6 & G9/1.5" & $2 \times 1200$ \\
$2003-02-28$ & EFOSC/3.6 & G4/1.5" & $3 \times 900$ \\
\hline
\end{tabular}

individual spectra and the photometry of the acquisition image and is estimated as $0.2 \times 10^{-18} \mathrm{~W} \mathrm{~m}^{-2} \mathrm{~nm}^{-1}$.

For the further analysis of both the photometric and spectroscopic data, the MIDAS package and self-written routines have been used.

\section{The method of recovery}

Cataclysmic variables are close, interacting binary systems, comprising a white dwarf accreting mass from a Roche-lobefilling late-type star. Hence there are at least three different physical components that provide the light observed from a cataclysmic variable. Due to its size and high temperature, the accretion disc or stream is generally the strongest in the optical range. While the white dwarf contribution affects mainly the UV range of the spectrum, the secondary late-type star contributes on the red and infrared side.

The compound of these different physical components results therefore in very characteristic colour terms. Cataclysmic variables appear as generally very blue objects with a shift towards the red at longer wavelengths, depending on the strength of the secondary in comparison to the accretion disc or stream. In a colour-colour diagram, this quality places them on the blue side but slightly above the main sequence.

Performing multi colour photometry on all stars within the field of a suspected nova should therefore show the following picture: the majority of the stars are gathered along a main sequence. The latter is shifted from the theoretical one according to the mean interstellar reddening in the field, while the scatter of the stars indicates the variation of the extinction within the field. All candidates within the coordinate uncertainty of the nova will be marked. Assuming that the interstellar reddening of the nova is of the same order as the other field stars, it should be located on the blue side above the main sequence defined by the field stars, and can thus be identified.

\section{Results and discussion}

\subsection{The nova candidate}

Figure 1 shows a $1 \times 1$ arcmin close-up of the $B$ image surrounding the nova position. The three stars that lie within the uncertainty range of the nova coordinates are indicated by the same

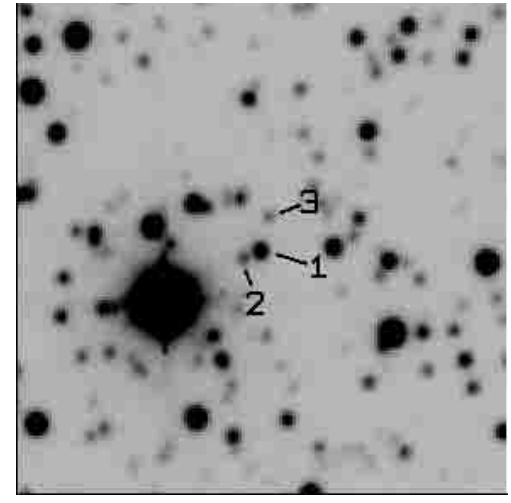

Fig. 1. The $B$-band finding chart shows the $1 \times 1$ arcmin surroundings of V840 Oph; north is up, east is left. The three possible candidates for the old nova are indicated by the numbers that have been assigned by Duerbeck (1987).

ID numbers as in Duerbeck (1987). In Table 2 the magnitudes and colours of these three objects are given. Figure 2 gives the colour-colour diagrams of all stars in the field with the three candidates marked with their numbers. The field stars define the main sequence, shifted and spread due to individual interstellar reddening effects. The mean extinction in the field has been determined as $E_{B-V}=0.4 \pm 0.15$. Whereas candidates 2 and 3 lie within the bulk of field stars, candidate 1 clearly stands out in these diagrams.

To verify our method, we took a first spectrum of this best candidate. The result is plotted in Fig. 3. In spite of the low $\mathrm{S} / \mathrm{N}$, the Balmer emission lines, typical for cataclysmic variables, are clearly visible and thus confirm the photometric selection. In addition, the nova character of this candidate is supported by the presence of He II $\lambda 5412$, since this line is typically found in nova remnants but not in dwarf novae or nova-like variables (Warner 1995). We therefore conclude that candidate 1 is indeed the old nova V840 Oph.

\subsection{The carbon content}

The strong line of CIV at $\lambda=580.5 \mathrm{~nm}$ is very rare in cataclysmic variables. CIV is usually detected in the hot wind of Wolf-Rayet stars, in planetary nebulae or during nova outburst and is there assumed to originate from the expanding shell. In the case of V840 Oph, however, the principal indicator for ongoing outflow, emission lines with a PCyg profile, is not observed. Furthermore, too much time has passed since the nova outburst in 1917 for the nova shell to provide a significant contribution, especially as it has been a very fast nova.

Although uncommon, CIV has been found before in quiescence novae like CP Pup (Williams \& Ferguson 1983), V603 Aql, V1500 Cyg, or HR Del (all in Ringwald et al. 1996), in the nova-like variables PG 1012-03 (Williams \& Ferguson 1983) and V Sge (Williams 1983), and most recently in QU Car (Drew et al. 2003). However, in all these systems the C IV line $\lambda 580 / 1$ appears much weaker, the strongest one being the line in QU Car with an equivalent width of $0.13 \mathrm{~nm}$, which is still a factor of five smaller than our detection in V840 Oph. However, we have to consider this number with caution, since all 
Table 2. Position, magnitudes and colours for the three candidates are given with their photometric errors. The ID numbers are as in Fig. 1.

\begin{tabular}{cccccccc}
\hline \hline ID & $\mathrm{RA}_{2000}$ & $\operatorname{Dec}_{2000}$ & $V$ & $U-B$ & $B-V$ & $V-R$ & $V-I$ \\
\hline 1 & $16: 54: 43.92$ & $-29: 37: 26.8$ & $19.32(3)$ & $-0.72(2)$ & $-0.21(3)$ & $0.40(3)$ & $0.99(4)$ \\
2 & $16: 54: 44.07$ & $-29: 37: 27.7$ & $19.71(3)$ & $0.43(6)$ & $0.77(4)$ & $0.40(4)$ & $1.34(5)$ \\
3 & $16: 54: 43.85$ & $-29: 37: 22.9$ & $19.95(3)$ & $0.27(7)$ & $0.97(4)$ & $0.98(4)$ & $1.41(5)$ \\
\hline
\end{tabular}
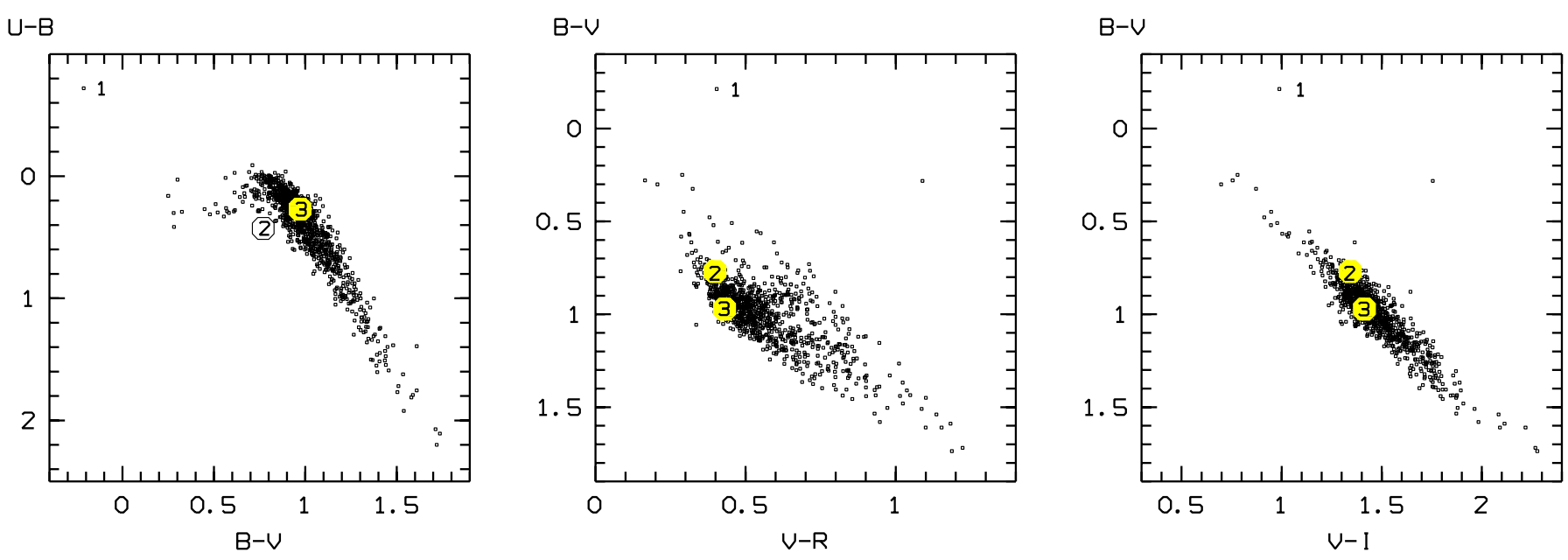

Fig. 2. Colour-colour diagrams are plotted for the three nova candidates and the surrounding field stars. While candidates 2 and 3 lie close to the main sequence (defined by the majority of field stars), the best candidate (No. 1) clearly stands out in all three diagrams.

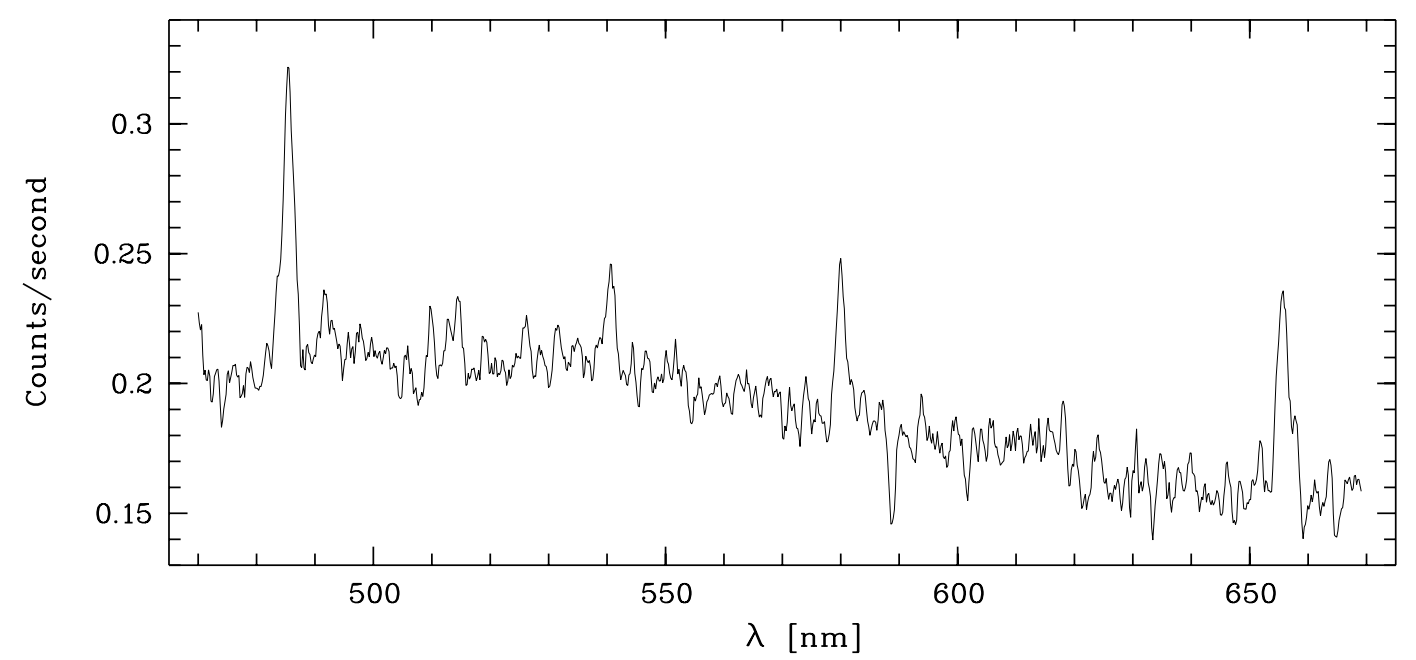

Fig. 3. Spectrum of candidate No. 1. Its nova character is confirmed by the presence of typical nova emission lines. Noteworthy are the strong He II line at $\lambda=541 \mathrm{~nm}$ and the presence of the C IV doublet at $\lambda=580 / 1 \mathrm{~nm}$ (not resolved).

emission lines in V840 Oph have a two to three times higher equivalent width than in QU Car, which can easily be explained by QU Car having a hotter continuum. Even taking this into account, the strength of the CIV line in V840 Oph is still remarkable.

Since C II and C III are common in most cataclysmic variables, there exist two possible scenarios, which can explain the presence of a strong C IV emission in V840 Oph:

(1) The temperature of V840 Oph or in parts of this system is extremely high, with the result that most of the carbon present is actually ionised to $\mathrm{C}^{4+}$, and the observed C IV emission line results from the $\mathrm{C}^{4+} \rightarrow \mathrm{C}^{3+}$ recombination cascade.

(2) The total amount of carbon in V840 Oph is higher than usual in these systems yielding corresponding high abundances for all carbon ions.

The first scenario would show in rather weak C II and C III lines and corresponding high ionisation ratios of CIV/CII and CIV/CIII. The second scenario would show in extremely strong C II and C III lines to keep the ionisation ratios on a normal level in spite of the strong C IV line. Naturally, a combination of both effects seems possible. 


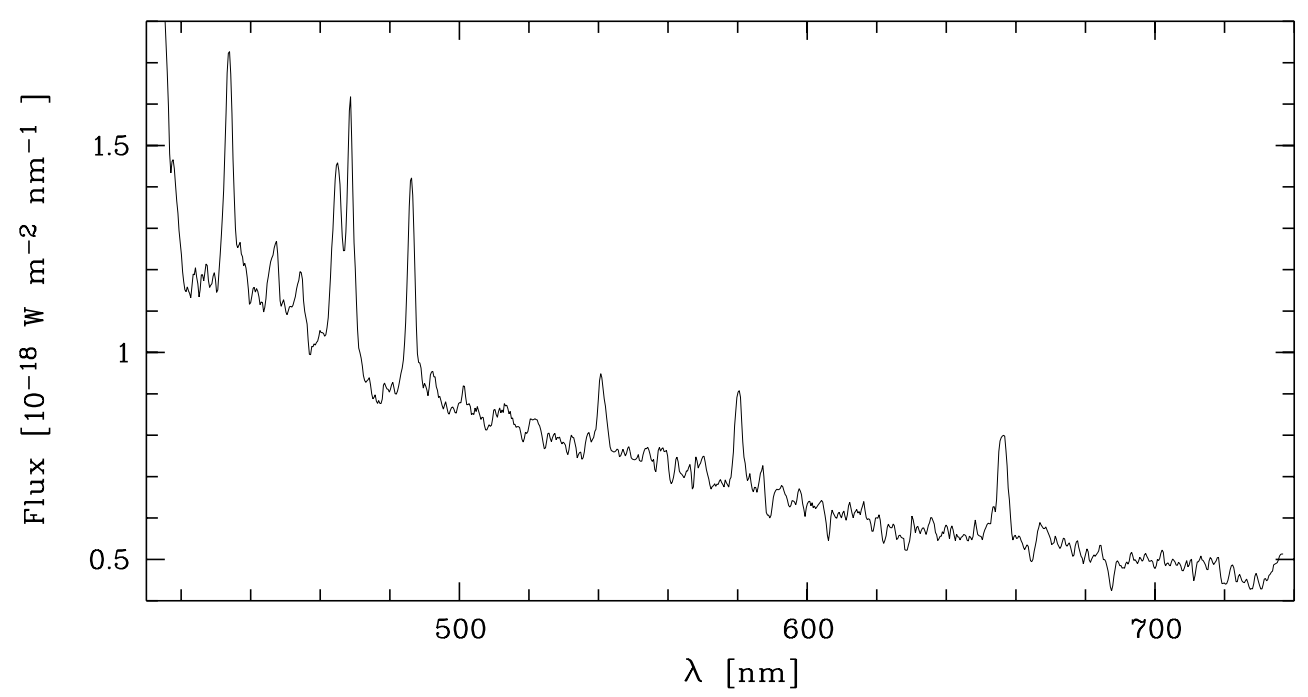

Fig. 4. Flux calibrated spectrum of V840 Oph (candidate 1), obtained in February 2003. The spectra has not been corrected for reddening. Apart from the high excitation lines already seen in the 2002 spectrum, the strong emission feature between $\lambda=460$ and $475 \mathrm{~nm}$ (Bowen blend and $\mathrm{He}$ II) attracts attention.

Table 3. Central wavelength and line flux as measured and dereddened (same units) are given of the fitted lines and blends for all ions within the $460-472 \mathrm{~nm}$ emission feature.

\begin{tabular}{lccc}
\hline \hline Ion & $\lambda$ & $F\left[10^{-18} \mathrm{~W} \mathrm{~m}^{-2}\right]$ & dereddened \\
\hline He II & 468.67 & $1.46 \pm 0.1$ & $4.03 \pm 0.3$ \\
C IV & 465.80 & $0.39 \pm 0.5$ & $1.60 \pm 2.0$ \\
C III & 464.95 & $0.68 \pm 0.2$ & $2.78 \pm 0.8$ \\
N III & 463.91 & $0.47 \pm 0.5$ & $1.92 \pm 2.0$ \\
O II & 465.43 & $0.10 \pm 0.5$ & $0.40 \pm 2.0$ \\
\hline
\end{tabular}

To check on these possibilities, we have taken an additional spectrum in the range 410 to $740 \mathrm{~nm}$, which also includes the Bowen blend (C III, C IV, N III) at $464 \mathrm{~nm}$ (see Fig. 4). Since the transmission was stable during this night, we performed a rough flux calibration for this spectrum. We used the photometrically determined reddening of $E_{B-V}=0.4$ to deredden the spectrum. However, the resulting continuum slope of $\lambda^{-3.3}$ seems to be quite high. Since the nova as an intrinsically faint object might be closer than the average of the field stars, and the scatter in the colour-colour diagram and hence in the derived extinction is quite high, we assume that $E_{B-V}=0.4$ is an upper limit for the nova extinction with the real value being probably lower. Hence, as a convention we have listed both flux values - the ones as observed and the dereddened ones in the tables. In the further text, however, we refer to the values as observed, if not stated otherwise.

We have modelled the Bowen emission feature by fitting Gaussian profiles to the individual lines of the four ion multiplets O II (8 lines), NIII (3 lines), CIV (1 line), and C III (3 lines) using the relative laboratory line strengths and wavelengths from McClintock et al. (1975) for the components within each multiplet. A common line width has been assumed for the individual lines. Its value and the four heights of the combined lines within one multiplet are the five parameters to fit. Since He II at $468.6 \mathrm{~nm}$ is not completely separated from

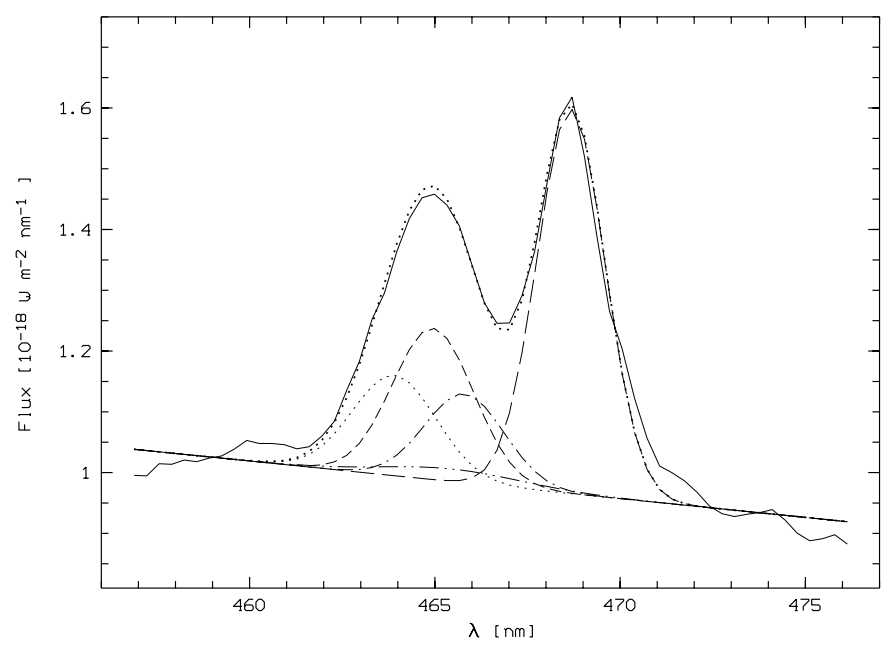

Fig. 5. A detailed view on the Bowen blend and He II $\lambda 469$. Over the original spectrum the fits to the five ions are plotted as dotted line for N III, dashed line for C III, dash-dot line for C IV, dash-dot-dot line for O II, and long dashes for He II. The overall fit is plotted as thick dots.

the blend, we have included a fit to the He II part, keeping all three Gaussian parameters free for this line. With the resulting best fit values we rebuilt the multiplets of each ion separately to compute its $F W H M$ and the flux contribution within. The results of these line-models are shown in Table 3 and Fig. 5. The $F W H M$ of the individual lines resulted in $2.5 \pm 0.8 \mathrm{~nm}$ which is in good agreement with the average measured for the other lines in the spectrum (see Table 4).

Other evidence for the goodness of the fit comes from the line strengths of C III and CIV. Both ions have a relatively strong line in the observed part of the spectrum, additionally to their contribution to the Bowen blend. The ratio of effective recombination coefficients for C IV $\lambda 466$ and C IV $\lambda 580 / 1$ is 1.67 (from Kingsburgh 1995). Scaling the C IV $\lambda 580 / 1$ line accordingly, one would expect a flux contribution of $0.4 \times$ $10^{-18} \mathrm{~W} \mathrm{~m}^{-2}$ of CIV to the Bowen blend, which is in 
Table 4. Equivalent widths, and FWHM of all identified emission lines in the 2002 and 2003 spectrum of V840 Oph. For 2003 also the line flux as measured and dereddened (same units) is given. Note that the uncertainty of the line flux describes the uncertainty of the relative flux in the line and does not include the photometric error.

\begin{tabular}{lcccccc}
\hline \hline & \multicolumn{2}{c}{ April 2002} & \multicolumn{4}{c}{ February 2003} \\
Transition & $F W H M[\mathrm{~nm}]$ & $-W[\mathrm{~nm}]$ & $F W H M[\mathrm{~nm}]$ & $-W[\mathrm{~nm}]$ & $F\left[10^{-18} \mathrm{~W} \mathrm{~m}^{-2}\right]$ & dereddened \\
\hline $\mathrm{H}_{\alpha}$ & $2.44 \pm 0.03$ & $1.55 \pm 0.15$ & $3.18 \pm 0.03$ & $1.66 \pm 0.05$ & $0.92 \pm 0.04$ & $2.28 \pm 0.10$ \\
$\mathrm{H}_{\beta}$ & $2.15 \pm 0.08$ & $1.35 \pm 0.12$ & $2.45 \pm 0.05$ & $1.51 \pm 0.06$ & $1.37 \pm 0.04$ & $5.22 \pm 0.15$ \\
$\mathrm{H}_{\gamma}$ & & $2.63 \pm 0.08$ & $1.58 \pm 0.06$ & $1.84 \pm 0.04$ & $8.30 \pm 0.18$ \\
He I $\lambda 668$ & & $3.20 \pm 0.50$ & $0.27 \pm 0.10$ & $0.15 \pm 0.10$ & $0.36 \pm 0.24$ \\
He I $\lambda 588^{*}$ & & $<0.1$ & $>1.50$ & $>0.12$ & $>0.08$ & \\
He I $\lambda 447$ & & & $2.47 \pm 0.08$ & $0.38 \pm 0.05$ & $0.42 \pm 0.03$ & $1.82 \pm 0.13$ \\
He II $\lambda 541$ & $1.74 \pm 0.07$ & $0.4 \pm 0.1$ & $2.55 \pm 0.04$ & $0.61 \pm 0.05$ & $0.45 \pm 0.03$ & $1.43 \pm 0.09$ \\
He II $\lambda 469^{* *}$ & & & $2.16 \pm 0.15$ & $1.50 \pm 0.07$ & $1.45 \pm 0.05$ & $5.84 \pm 0.20$ \\
He II $\lambda 454$ & & & $2.47 \pm 0.08$ & $0.37 \pm 0.05$ & $0.40 \pm 0.04$ & $1.69 \pm 0.17$ \\
C III $\lambda 570$ & & $<0.1$ & $2.57 \pm 0.30$ & $0.30 \pm 0.04$ & $0.21 \pm 0.02$ & $0.62 \pm 0.06$ \\
C IV $\lambda \lambda 580 / 1$ & \multirow{2}{*}{$1.71 \pm 0.02$} & $0.7 \pm 0.1$ & $2.46 \pm 0.04$ & $0.96 \pm 0.03$ & $0.65 \pm 0.02$ & $1.89 \pm 0.06$ \\
Bowen blend ** & & & $3.21 \pm 0.15$ & $1.64 \pm 0.07$ & $1.64 \pm 0.05$ & $6.70 \pm 0.20$ \\
\hline
\end{tabular}

* This He I line is disrupted by the nearby $\mathrm{Na}$ absorption line.

** The properties have been derived by fitting five ion components to these blended lines (see text and Fig. 5).

perfect agreement with our results (Table 3). For C III, the ratio of C III $\lambda 570$ flux and C III $\lambda 465$ flux from laboratory spectra is 0.32 . Scaling the fitted value of $F=0.68 \times 10^{-18} \mathrm{~W} \mathrm{~m}^{-2}$ accordingly, the flux in C III $\lambda 570$ is expected around $0.22 \times$ $10^{-18} \mathrm{~W} \mathrm{~m}^{-2}$, which is within the errors of the found value (see Table 4). For the N III contribution, no such confirmation exists, since all other strong N III multiplets are blended with either $\mathrm{H} \beta$, He II, or $\mathrm{H} \gamma$. At $451 \mathrm{~nm}$ a small emission is seen but the noise in this part of the spectrum is too high for a trustworthy detection of this weak feature. Small peaks are also found for the N III multiplet around $646 \mathrm{~nm}$ but the identifications are dubious due to blending with several Fe II multiplets in this region. The low contribution of O II to the blend is confirmed by the absence of features at $459.3 \mathrm{~nm}$ and $470.5 \mathrm{~nm}$.

The total flux in the Bowen blend sums up to $1.64 \times$ $10^{-18} \mathrm{~W} \mathrm{~m}^{-2}$, which is slightly higher than the flux of He II at $468.6 \mathrm{~nm}$. So far, QU Car has been the only known cataclysmic variable where the emission from the Bowen blend is comparable to the He II emission at $468.6 \mathrm{~nm}$. In V840 Oph, about two thirds of this flux are contributed by carbon emission with C III being about twice as strong as C IV. C III and N III contribute in a ratio of about 3 to 2 , while the OII emission is negligible. This appearance is similar to what has been found in QU Car (Gilliland \& Phillips 1982) with nitrogen being slightly more pronounced in V840 Oph. Accordingly, fluorescence via the Bowen mechanism can probably be discarded as major excitation mechanism, whereas an enhancement of the $\mathrm{CN}$ abundance seems more likely to explain the strength of this feature.

Although high, the ratio of CIV $\lambda \lambda 580 / 1$ to the nearby He II $\lambda 541$, which is 1.5 for V840 Oph, is lower than the 3.5 measured for QU Car (Drew et al. 2003) and V840 Oph might hence be more similar to V Sge where a ratio of 1.3 has been found by Herbig et al. (1965) although values of 0.4 have also been reported (Williams 1983). However, for V840 Oph, this ratio is certainly affected by the extremely strong He II $\lambda 541$ line which in V840 Oph is only a factor three fainter than He II 2468 . Comparing instead the ratio C IV $\lambda \lambda 580 / 1$ to He II $\lambda 468$ we get 0.5 for V840 Oph which is slightly higher than for QU Car.

To better understand the abundances of the different ions and their excitation mechanism, the study of UV-spectra will be necessary. Additional time-resolved spectroscopy of the C IV and C III lines could help to clarify the location of the carbon emission source within the system.

So far, one can certainly conclude that the strength of the Bowen blend as well as the strength of He II hints towards a higher than solar metallicity in the accreted matter. Therefore the secondary in V840 Oph must be an evolved star. Hence, the overabundance of carbon, similar to QU Car, can be similarly explained by assuming a secondary carbon star.

Further evidence for a carbon star secondary might come from signatures of the secondary in the spectrum of V840 Oph. To search for these features, we have fitted a $\lambda^{\alpha}$ power law to the blue part of the spectrum, leaving out the line features. For the dereddened spectrum, we derive $\alpha=3.303$ (3). After subtracting this slope from the spectrum we derive the flat spectrum plotted in Fig. 6. Only a slight enhancement is found at the red end that could be due to the red continuum of the secondary but might also be and artificial fit residual. Three absorption features ( $\mathrm{Na}$ and two unidentified bands as marked in Fig. 6) are found that are also present in the 2002 data and that we hence concluded to be real, although the two bands are just visible above the noise and we cannot derive their strength. Furthermore, the Na line might partly be interstellar. However, if all the $\mathrm{Na}$ was interstellar, its observed equivalent width 


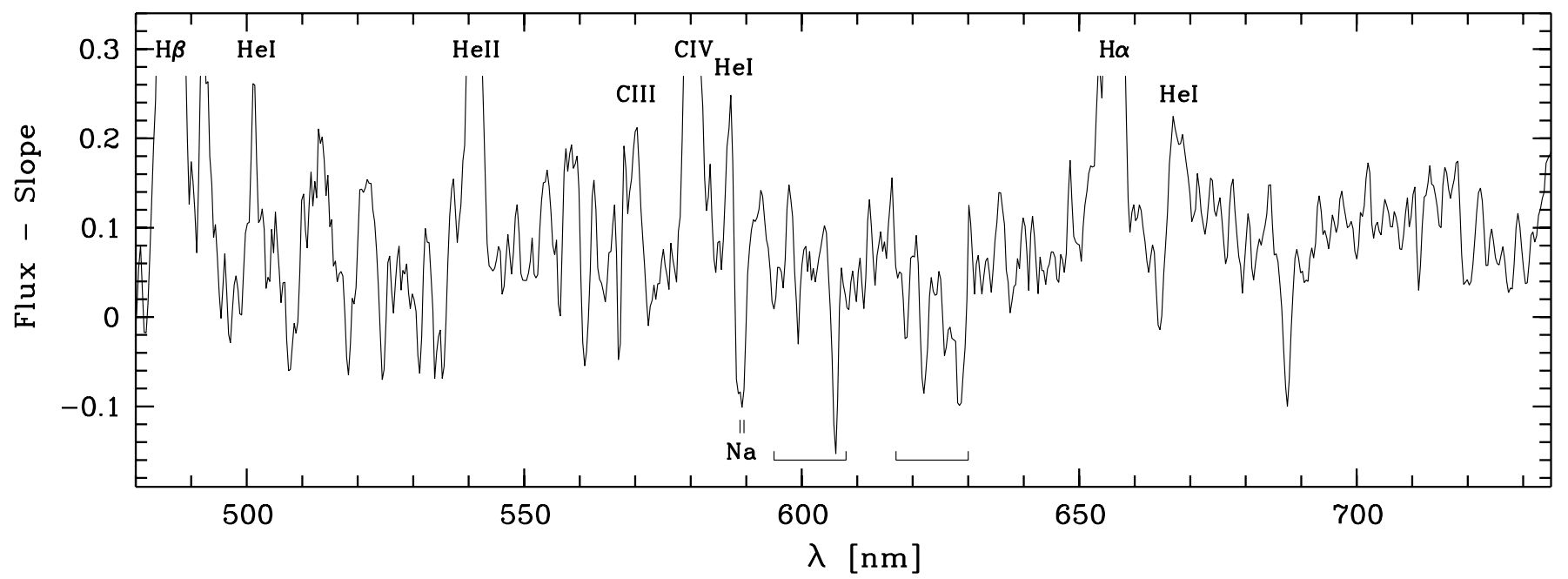

Fig. 6. Result of subtracting a $\lambda^{-3.3}$ slope from the dereddened spectrum of V840 Oph. The identified emission lines and those absorption features that are also present in the 2002 data are marked.

of $0.19(4) \mathrm{nm}$, which can be considered as a lower limit since the line is partly filled up by the nearby He I 587.6 emission, would yield an extinction of $E_{B-V}>1.7$ (Zwitter \& Munari 1998) which is certainly too high. To match the photometrically derived reddening of $E_{B-V}=0.4$, at least half of the equivalent width has to be due to stellar absorption.

In an attempt to match the absorption features we find that late type $\mathrm{K}$-stars as well as $\mathrm{M}$-stars show deep TiO bands with respect to the features present in our data which then should be visible in the red part of the spectrum. Very early type K-stars as well as G-stars do not have such a pronounced $\mathrm{Na}(589 \mathrm{~nm})$ doublet as to account for what is observed in the spectrum of V840 Oph. Although spectroscopic observation in the NIR are needed to improve the classification, we tentatively conclude that the secondary is best represented by a mid-type $\mathrm{K}$-star. This is comparable to what has been found for QU Car, where a late $\mathrm{R}$-star has been suggested as secondary (Drew et al. 2003).

\subsection{Other results from the spectral analysis}

In Table 4, the equivalent width and FWHM in both data sets are listed for all identified emission lines. For the 2003 data also the line fluxes as observed and dereddened with $E_{B-V}=0.4$ are given. With values around $2.0 \mathrm{~nm}$, the $F W H M$ is quite narrow for a cataclysmic variable. This might indicate that the system is seen at a low inclination.

A rough comparison of the features already reveals an interesting finding: the equivalent widths of all lines common in both spectra are systematically higher in the 2003 data than in 2002. Furthermore, although faint, He I is present in this spectrum at $\lambda=588 \mathrm{~nm}$ and $\lambda=668 \mathrm{~nm}$ which were not detected in 2002. Both these facts indicate that either V840 Oph was in a higher accretion state during the 2002 observation compared to 2003 , or there is a dependence of the spectral features on the orbital phase. With the present data we can not decide between these possibilities.
In order to derive information on the possible temperature range of V840 Oph, we have measured the Balmer decrement, which is defined as ratio of line intensities $\mathrm{H}_{\alpha}: \mathrm{H}_{\beta}: \mathrm{H}_{\gamma}$. We have computed the ratios of line fluxes of $\mathrm{H}_{\alpha}$ and $\mathrm{H}_{\gamma}$ over $\mathrm{H}_{\beta}$ for the 2003 data set and derive $\mathrm{H}_{\alpha} / \mathrm{H}_{\beta}=0.67$ ( 0.45 dereddened) and $\mathrm{H}_{\gamma} / \mathrm{H}_{\beta}=1.34$ (1.59). Allowing for the fact that the reddening might be over-corrected, the uncorrected data still yield a limit for the Balmer decrement and an accordingly lower limit for the temperature and density. This highly inverted Balmer decrement indicates that the line emission has its origin in a hot and dense environment. A comparison of the lines as observed (without reddening correction) with model data from Williams (1991) shows indeed that the derived values do not fit in his grid, which is computed for temperatures up to $15000 \mathrm{~K}$, but that an extrapolation towards higher temperatures above $30000 \mathrm{~K}$ is necessary to match both ratios and the equivalent widths of the lines. Similar high temperatures are necessary to explain the strength of He II compared to the lack of $\mathrm{He}$ I. At least $20000 \mathrm{~K}$ are estimated for the line-emitting region to maintain the He ionisation.

The presence of high excitation lines and especially the high ratio $\mathrm{HeII} \lambda 468 / \mathrm{H} \beta \approx 1.0$, the absence of $\mathrm{He} \mathrm{I}$ in the 2002 data and its weakness in 2003, and the visibility of absorption lines of the secondary, suggest V840 Oph to be a magnetic system with no or only a very weak disc. This is supported by the inverted Balmer decrement which also points towards a magnetic classification (see e.g. Schachter et al. 1991; Ferrario \& Wehrse 1999). However, the lack of any pronounced cyclotron lines in the observed part of the spectrum indicates that the magnetic field can only be of moderate strength.

\section{Summary}

Using multi colour diagrams for a first selection and optical spectroscopy for the final confirmation we could identify the classical nova V840 Oph with an object of $V=19.32$ at the position $\alpha=16^{\mathrm{h}} 54^{\mathrm{m}} 43^{\mathrm{s}} .9, \delta=-29^{\circ} 37^{\prime} 27^{\prime \prime}$ (J2000). 
The spectrum of V840 Oph contains an unusually strong C IV emission at $\lambda=580 / 1 \mathrm{~nm}$. Furthermore the Bowen blend is strong and carbon-dominated. We have concluded that similar to QUCar, the spectral features are best explained by assuming a carbon star as secondary.

V840 Oph is probably a low inclination system. This highly inverted Balmer decrement and the presence of high excitation lines suggest the system to have extremely high temperatures above $30000 \mathrm{~K}$. We furthermore find spectroscopic similarities to magnetic old novae, suggesting a corresponding scenario for V840 Oph. More detailed investigations of this system are desirable to confirm its nature.

Acknowledgements. We would like to thank George Hau and Michael Sterzik, who kindly performed the spectroscopic observations of V840 Oph in 2002, Robert Schwarz for information on magnetic CVs, and the referee Janet Drew for helpful comments. We acknowledge that this research has made use of the Simbad database operated at CDS, Strasbourg, France.

\section{References}

Bailey, S. I. 1920, AN, 210, 375

McClintock, J. E., Canizares, C. R., \& Tarter, C. B. 1975, ApJ, 198, 641
Drew, J. E., Hartley, L. E., Long, K. S., \& van der Walt, J. 2003, MNRAS, 338, 401

Duerbeck, H. W. 1981, PASP, 93, 165

Duerbeck, H. W. 1987, Space Sci. Rev., 45, 1

Ferrario, L., \& Wehrse, R. 1999, MNRAS, 310, 189

Gilliland, R. L., \& Phillips, M. M. 1982, ApJ, 261, 617

Harrison, T. E., \& Gehrz, R. D. 1994, AJ, 108, 1899

Herbig, G. H., Preston, G. W., Smak, J., \& Paczynski, B. 1965, ApJ, 141,617

Hoard, D. W., Wachter, S., Clark, L. L., \& Bowers, T. P. 2002, ApJ, 565,511

Horne, K. 1986, PASP, 98, 609

Kingsburgh, R. L., Barlow, M. J., \& Storey, P. J. 1995, A\&A, 295, 75

Landolt, A. U. 1992, AJ, 104, 340

Munari, U., \& Zwitter, T. 1998, A\&AS, 128, 277

Ringwald, F. A., Naylor, T., \& Mukai, K. 1996, MNRAS, 281, 192

Schachter, J., Filippenko, A. V., Kahn, S. M., \& Paerels, F. B. S. 1991, ApJ, 373, 633

Shapley, H. 1921, PASP, 33, 189

Warner, B. 1995, Cataclysmic Variable Stars (Camebridge University Press)

Williams, G. 1983, ApJS, 53, 523

Williams, G. A. 1991, AJ, 101, 1929

Williams, R. E., \& Ferguson, D. H. 1983, in Cataclysmic Variables and Related Objects, ed. Livio, \& Shaviv, IAU Coll., 72, 114

Zwitter, T., \& Munari, U. 1998, in Wild Stars in the Old West, ed. S. Howell, E. Kuulkers, \& C. Woodward, ASP Conf. Ser., 137, 35 